le portiQue $\begin{array}{ll}\text { Le Portique } \\ \text { Revue de philosophie et de sciences humaines }\end{array}$

$10 \mid 2002$

Les paradis artificiels

\title{
La muse au corps à corps
}

\section{Michèle Kuntz}

\section{(2) OpenEdition}

Journals

Édition électronique

URL : http://journals.openedition.org/leportique/144

DOI : 10.4000/leportique.144

ISSN : $1777-5280$

\section{Éditeur}

Association "Les Amis du Portique"

Édition imprimée

Date de publication : 1 septembre 2002

ISSN : 1283-8594

\section{Référence électronique}

Michèle Kuntz, "La muse au corps à corps », Le Portique [En ligne], 10 | 2002, mis en ligne le 06 juin 2005, consulté le 09 avril 2021. URL : http://journals.openedition.org/leportique/144 ; DOI : https:// doi.org/10.4000/leportique.144

Ce document a été généré automatiquement le 9 avril 2021.

Tous droits réservés 


\title{
La muse au corps à corps
}

\author{
Michèle Kuntz
}

1 La drogue est un objet littéraire lorsque, passant par le corps, elle resurgit dans l'imaginaire pour se déposer dans l'écriture en pensées et en images.

2 J'ai fait le choix, non pas subjectif mais, au contraire, très surdéterminé de deux époques où la drogue noua des liens serrés avec la littérature. Il s'agit du XIX ${ }^{\mathrm{e}}$ siècle, qui vit se rencontrer, autour de la drogue, scientifiques et littéraires; qui vit aussi naître une œuvre littéraire majeure, Les Paradis artificiels de Baudelaire. Il s'agit de la beat generation des années 50, dans laquelle quelques écrivains s'engagèrent tout en livrant, dans leurs œuvres, d'irremplaçables éléments quant à la compréhension du lien qui unit une personne à une drogue.

3 Parce que l'espace où se joue le lien avec la drogue est le corps, et parce que l'inspiratrice des œuvres que je vais évoquer est la drogue elle-même, j'ai intitulé ma communication : «La Muse au corps à corps ».

4 Avant d'entrer dans le vif de cette lutte entre expérience de consommation de drogue et création littéraire, j'évoquerai les milieux culturels où la drogue comptait parmi les invités.

Le club des haschichins

5 Dans ce que j'appellerai le $x_{x}{ }^{e}$ siècle scientifique et littéraire, la drogue voyage entre science et littérature. La morphine est isolée en 1817 ; en 1821 paraissent Les Confessions d'un mangeur d'opium anglais de Thomas de Quincey. En 1828, l'œuvre est traduite en français par un auteur anonyme ADM, qui s'avère être Musset, tandis que, dans la foulée, une thèse de médecine sur l'usage de l'opium porte en exergue la fameuse formule de de Quincey : « Ô juste, subtil et puissant opium ».

6 Entre 1837 et 1840, le médecin aliéniste Moreau de Tours consomme du haschich au gré de ses voyages - en Égypte, Syrie, Asie Mineure. De retour en France, il continue à l'expérimenter sur lui-même et publie, en 1845, un ouvrage intitulé Du haschich et de l'aliénation mentale dans lequel il établit une équivalence entre rêve, délire et hallucinations haschichines. 
7 De ces dates qui jalonnent la première moitié du $\mathrm{xIX}^{\mathrm{e}}$ siècle, on peut dire qu'elles marquent l'intérêt conjoint de la science et de la littérature pour les drogues. On remarquera aussi qu'à les relier, se dessinent deux types de voyages: un voyage géographique entre France, Angleterre et Moyen Orient ; un va-et-vient entre science et littérature.

Lorsque après le milieu du siècle, Baudelaire entreprend Les Paradis artificiels, il voyage, quant à lui, entre ses propres expériences du vin, du haschich et l'expérience d'un autre, le mangeur d'opium anglais, revisitée par lui-même. De cet alliage - le terme est scientifique - de cette alliance - le terme est culturel - naîtra une œuvre dont le titre est passé dans le vocabulaire usuel.

9 Cette échappée des drogues dans les milieux de vie s'illustre, au milieu du siècle, dans le club des haschichins. Créé par Moreau de Tours en 1845, il accueillit des scientifiques, des hommes de lettres et des artistes, au nombre desquels on compta le célèbre aliéniste Esquirol, Balzac, Baudelaire, Gautier, Nerval, Daumier. Il dut, sans doute, une partie de son succès à sa situation exceptionnelle, dans un hôtel particulier de l'île Saint-Louis, l'hôtel de Lauzun, encore appelé hôtel Pimodan. Mais ce qui constitua sont originalité furent les séances de drogue, encore appelées fantasias. Des conversations, des auditions musicales firent aussi partie des habitudes du club.

On sait que Moreau de Tours initia Gautier, et cela bien avant la création du club puisque, dans un feuilleton daté de juillet 1843 et intitulé «Le Haschich», Gautier relate les effets de la drogue en différenciant trois phases: hyperesthésie des sensations, en particulier auditives, dilatation du temps, enfin apparition de figures grotesques.

11 En février 1846, Gautier consacre un opuscule à la relation d'une séance de drogue au club, mais l'année 1846 voit surtout l'exploitation littéraire des hallucinations dues au haschich dans un texte où tous les sens sont convoqués :

12 «Au bout de quelques instants, j'éprouvai à l'estomac une légère chaleur, mon corps jetait des étincelles et brûlait comme un billet de banque à la flamme d'une bougie. [...] Tous mes sens étaient déplacés; je voyais la musique et j'entendais les couleurs [...] mon corps se composait, à mon gré, d'un rayon, d'un parfum ou d'une saveur ». Ce texte est extrait d'un roman, La Croix de Berny, écrit par Gautier en collaboration avec d'autres auteurs.

Balzac, lui aussi, fréquenta le club des haschichins. Le fait est attesté par Baudelaire dans Les Paradis artificiels et par Balzac lui-même. À les entendre l'un et l'autre, on remarquera que la relation de Balzac au haschich fut, pour le moins, ambiguë.

14 «Je l'ai vu une fois, dans une réunion [à Pimodan] où il était question des effets prodigieux du haschich», écrit Baudelaire, qui poursuit: "On lui présenta du dawamesk ; il l'examina, le flaira et le rendit sans y toucher... En effet, il est difficile de se figurer le théoricien de la volonté... consentant à perdre une parcelle de cette précieuse substance.»

15 Propos auxquels font écho ceux de Balzac lui-même lorsqu'il relate : "J'ai résisté au haschich et je n'ai pas éprouvé tous les phénomènes: mon cerveau est si fort qu'il fallait une dose plus forte que celle que j'ai prise. Néanmoins, j'ai entendu des voix célestes et j'ai vu des peintures divines. J'ai descendu pendant vingt ans l'escalier de Pimodan... Mais ce matin, depuis mon réveil, je dors toujours, et je suis sans volonté. » 

haschichines car, ainsi que l'écrit Baudelaire: "on vit plusieurs vies d'hommes en l'espace d'une heure ». Baudelaire qui poursuit: "c'est bien là le sujet de La Peau de chagrin». confrontant à l'imaginaire balzacien où «tous les excès sont frères " dès l'instant où Raphaël, le héros du roman, s'est saisi d'une peau d'âne sauvage, cette métaphore de la vie que les orgies - c'est un terme balzacien - rétrécissent en s'écriant : «Eh bien, oui, je veux vivre avec excès. »

18 Pour revenir à la réalité du club des haschichins, il faut dire que Baudelaire, dès le début de l'existence du club, le fréquenta en voisin puisqu'en 1845 il habitait l'étage audessus. Quant à l'implication de Baudelaire dans les activités du club, Gautier, dans sa préface des Fleurs du mal, indique qu'il fut surtout un observateur.

Au sein même de leur travail d'écriture, des écrivains ont donc noué des échanges. Dans ce milieu du xix siècle, le haschich fut plus qu'une curiosité de l'époque. Il fut un catalyseur, un médiateur.

Les Paradis artificiels

On retrouve cette fonction de médiateur de la drogue dans Les Paradis artificiels puisque l'œuvre regroupe des textes organisés, pour chacun d'eux, autour d'une ou de deux drogues. Par-delà son rôle de référence, la drogue est aussi, dans Les Paradis artificiels, prétexte à des comparaisons, à des correspondances. En dernière analyse, dans ces liens complexes tissés entre drogue et écriture, des trous laissent entrevoir l'impact corporel de la drogue.

21 Du vin et du hachisch, incorporé dans l'édition originale des Paradis artificiels de 1860, date de 1851. C'est dans le corps du texte qu'on trouve l'explicitation du titre : « Du vin et du hachisch comparés comme moyens de multiplication de l'individualité ».

22 Si «l'exaspération de la personnalité » - je cite encore Baudelaire - est à mettre sur le compte de chacune des deux substances, la comparaison entre vin et haschich est récurrente dans le texte: «Le hachisch procure une joie, un bien-être surabondants, mais il ne console pas comme le vin ».

Cette vertu consolatrice du vin est déjà présente dans un poème de 1850, L'Âme du vin : «Car j'éprouve une joie immense quand je tombe / Dans le gosier d'un homme usé par ses travaux ».

24 Elle est reprise avec entrain dans $D u$ vin et du hachisch: "je fais dans l'estomac du travailleur un grand remue-ménage, et de là, par des escaliers invisibles, je monte dans son cerveau où j'exécute ma danse suprême ».

25 Ainsi le vin voyage-t-il des sensations corporelles jusqu'à l'âme. Ainsi voyage-t-il aussi dans l'œuvre baudelairienne, et jusqu'à la préface de sa traduction des Nouvelles histoires extraordinaires d'Edgar Poe.

26 «Il prenait le chemin le plus dangereux mais le plus direct. Une partie de ce qui fait, aujourd'hui, notre jouissance est ce qui l'a tué », écrit Baudelaire à propos de Poe. La voie directe avec une substance - «il ne buvait pas en gourmand mais en barbare », dit encore Baudelaire - celle qui ne ménage en rien celui qui l'emprunte, est évoquée à travers un autre écrivain. 
27 Le Poème du hachisch constitue la seconde partie des Paradis artificiels. Baudelaire communique, déjà, la recette de la confiture verte, le dawamesk, telle que la préparent les arabes, indiquant, au passage, que cette préparation est bien plus efficace que l'herbe mêlée à du tabac.

Après avoir remarqué que l'ivresse due au haschich est « le naturel excessif... un miroir grossissant mais un pur miroir », Baudelaire met en scène des personnages sous l'effet de la drogue.

29 «Les sons se revêtent de couleurs et les couleurs contiennent une musique », cette conclusion d'une scène où "un littérateur» assiste à un spectacle intègre les transformations perceptives dues à la drogue dans le pôle sensoriel des correspondances.

30 Lorsque, chez un personnage fictif, un « esprit cultivé », le haschich « s'étend sur toute la vie comme un vernis magique; il la colore en solennité et en éclaire toute la profondeur ». Là, c'est le pôle spirituel des correspondances qui est en jeu.

31 Ainsi les deux axes des correspondances baudelairiennes, l'axe horizontal des sensations, l'axe vertical des effets sur l'âme sont-elles à l'œuvre dans la métamorphose littéraire des productions haschichines.

32 La fin du Poème du hachisch voit la retombée brutale de cette apothéose des sens et de l'esprit : «Mais le lendemain! Le terrible lendemain! [...] La hideuse nature, dépouillée de son illumination de la veille, ressemble aux mélancoliques débris d'une fête ».

33 "Ô juste, subtil et puissant opium!", " ces étranges actions de grâce » ainsi que les nomme Baudelaire, et qui figurent chez Thomas de Quincey à la fin d'un chapitre, inaugurent le texte final des Paradis artificiels, Un Mangeur d'opium. D'entrée de jeu, on sait qu'il ne s'agit pas d'une simple traduction.

34 Cette reprise du texte original se poursuit, Baudelaire intitulant le dénouement de de Quincey "un faux dénouement» pour conclure Les Paradis artificiels par un texte postérieur de de Quincey qui est consacré à son enfance. Baudelaire innove donc en matière de forme dramatique.

35 Il fait aussi ce qu'il nomme " un amalgame » entre ses sensations personnelles et les opinions de l'auteur original. Ainsi nous livre-t-il ce qu'il appelle une analyse. « Analyser ainsi c'est créer », dira Victor Hugo.

36 Baudelaire eut recours à l'opium. Sa correspondance, où il fait état de son renoncement - « j'ai pris tout excitant en horreur... impossible d'être un homme de lettres avec une orgie spirituelle continuée » - est, à cet égard, aussi éloquente que Les Confessions de de Quincey.

37 L'opium s'inscrit dans l'œuvre sous la forme d'un dialogue fictif entre les deux écrivains où chacun renverrait à l'autre les effets néfastes de la drogue sur la volonté.

38 «Il restait une préface à écrire (la fatigue d'une préface) », confie Baudelaire. Ce que de Quincey confirme, à propos d'un texte en préparation : "Ô humiliation d'un auteur nerveux, tyrannisé par l'atmosphère intérieure ». On retrouve l'opium, déposé dans la quintessence de l'écriture, dans un poème des Fleurs du mal, Le Poison.

40 On retrouve aussi cet amalgame entre les deux écrivains dont parle Baudelaire dans le retour sur l'enfance. Dans le dernier texte des Paradis artificiels est évoquée l'enfance de de Quincey, « cette sensibilité précoce qui fut pour lui la source de tant d'horreurs et de 
tant de jouissances ». Il faut se déporter du côté du Journal intime de Baudelaire pour y trouver un écho: "tout enfant, j'ai senti dans mon cœur deux sentiments contradictoires, l'horreur de la vie et l'extase de la vie». Parenté de sensibilité d'écrivains. Mise à distance dans l'écriture.

41 C'est encore par la médiation d'un autre écrivain, Edgar Poe, ou plutôt par le vocabulaire qu'il utilise - esclavage, chaînes de l'opium - que Baudelaire lâche cette phrase terrible : «épouvantable mariage de l'homme avec lui-même. » Cette sentence brutale, et qui tombe d'un coup, sans que rien n'y prépare le lecteur, sans qu'aucun éclaircissement ne lui soit ensuite donné, je l'entends comme une intuition exceptionnelle de l'écrivain quant à ce qu'il advient du psychisme - le désastre lorsque l'autre est éjecté. Dans ce que j'ai appelé «la muse au corps à corps », la muse n'est plus que le fantôme d'un corps privé de perspective.

La beat generation

Ce sont Les Paradis artificiels, titre du colloque et œuvre de Baudelaire parue en 1860 qui vont faciliter le passage vers le continent américain et la beat generation des années 50 .

«Il ne buvait pas en gourmand mais en barbare, avec une activité et une économie de temps tout à fait américaines ", écrivait Baudelaire à propos d'Edgar Poe. L' activité au sens de promotion de l'acte de la beat generation et la consommation de morphine «à l'américaine » dont l'écrivain William Burroughs fait état dans Le Festin $n u$, paru en 1959, sont, pour moi, deux nouveaux repères historiques. D'une manière différente parce que l'époque est autre, d'une manière comparable quant à la place qu'elle occupe, la drogue, ici encore, va et vient entre milieux de vie et littérature.

On a dit que la beat generation précédait le mouvement américain des années 60 , qui conjugue refus de la violence et libération des mœurs, et qui est très reconnu sous le nom de contre-culture. On a pu soutenir, à l'inverse, qu'elle en constituait le moment le plus original en raison de la cristallisation, dans quelques lieux new-yorkais, d'une vie culturelle intense et, parallèlement, d'un essaimage dans des voyages.

Joyce Johnson, qui fut la compagne de l'écrivain Jack Kerouac, formule ce type de jugement dans un roman, paru en 1983, qui fait le portrait des personnages principaux de la beat generation et recompose quelques atmosphères. Cet ouvrage, intitulé Personnages secondaires, pour bien marquer le rôle qui fut le sien et celui d'autres femmes de la beat generation, m'est apparu vivant et fiable parce que sans nostalgie ni rancœur.

L'esprit, l'atmosphère de la beat generation ont pu, selon Joyce Johnson, se concentrer dans quelques cafés de Greenwich Village. On y voyait des jeunes filles portant des jupes droites de tissu rêche tissé à la main, de longues boucles d'oreille de cuivre, des ceintures et des sandales en cuir enroulant leurs lacets autour des tailles et des chevilles. On y voyait des garçons dont la tenue - pull-over à col roulé, béret, lunettes noires - composait le personnage un peu dangereux du beatnik. On y parlait des noirs lynchés ou jetés en prison pour avoir sifflé, dans la rue, une femme blanche. On y entendait la mandoline d'un vieil italien à moustache blanche. On y buvait de l'espresso noir et amer et l'on fumait des cigarettes de marijuana qu'on se passait solennellement.

Dans Greenwich Village, le Waldorf, «l'équivalent des Deux Magots pour la $8^{\mathrm{e}}$ rue ", était, en 1950 et probablement depuis quelque temps, fréquenté par des écrivains et par des peintres. Un habitué du Waldorf eut, durant l'année 1950, je cite Joyce Johnson: 
«des conversations incohérentes mais étrangement inspirées avec un peintre alcoolique d'âge mûr répondant au prénom de Jackson ».

Il s'agissait de Jackson Pollock, l'un des chefs de file de l'école new-yorkaise de peinture qui devait consacrer New York comme un nouveau pôle de création picturale. Il n'est pas inutile, ici, d'évoquer la stature de Pollock, créateur de l'action painting, la peinture dans l'action de peindre. D'évoquer aussi cette révolution du geste pictural où c'est une danse frénétique autour de la toile qui produit l'œuvre, le rythme du tableau répondant à la pulsation de la vie de l'artiste. Pollock devait, après une courte période de fortune et de gloire, terminer sa vie en traversant un pare-brise à 160 à l'heure.

Autre lieu new-yorkais: l'appartement de six pièces de la $115^{\mathrm{e}}$ rue qu'occupaient l'écrivain William Burroughs et sa femme Joan. C'était - je cite Joyce Johnson - « un appartement légendaire... car il y avait certainement quelque vérité cachée au fond de ce trouble univers de dealers et de camés, de voleurs et de putains. » Burroughs était l'héritier d'une société qui commercialisait une machine à calculer inventée par son grand-père. Il possédait une mitraillette et vendait de la morphine. Il partageait avec Joan le goût des œuvres de Kafka et des drogues. Le personnage de Joan devait s'effacer brutalement parce qu'un soir de 1951, dans un geste insolent et peut-être suicidaire, elle mit son verre au sommet de sa tête et que William, pourtant tireur d'élite, visa mal ce soir-là.

La beat generation sollicita les réactions des critiques littéraires. Dans le Sunday Times du 16 novembre 1952, on pouvait lire sous le titre «Voici la beat generation »: «c'est un désir inassouvi de paroles, de joie, d'excitation, de sensations, de vérités nouvelles ».

51 Joyce Johnson apporte, rétrospectivement, sa propre définition lorsqu'elle rapproche «les entrelacs vertigineux de peinture appliquée directement à partir du tube » de Pollock, un chorus de Charlie Parker et la prose de Kerouac, « autre danse dans le flux du temps ».

52 Il me semble, quant à moi, que ce "désir inassouvi », c'est-à-dire cette urgence qui s'accroche et qui n'en démord pas, c'est le beat, le rythme.

53 Le roman de Joyce Johnson prend appui sur une photographie prise à l'université Columbia de New York où l'on voit côte à côte William Burroughs, Jack Kerouac et Allen Ginsberg. Ginsberg rédigea un journal au fil de ses voyages, écrivit des poèmes. Un fragment de son journal relatif à l'année 1954 le montre voyageant dans le Yucatan.

54 À Chichen Itza, « il plane à la paracodéine, allongé dans un hamac devant les grandes ruines d'El Castillo ». Lorsqu'il passe la frontière qui le ramène en Californie, il note : « entre États-Unis seul, nu, avec sac, montre, appareil-photo, poème, barbe. »

55 En 1956, à la Six Gallery à San Francisco, Ginsberg donne une lecture de son poème, Hurlement. Plus exactement, il réalise une performance associant plusieurs genres artistiques et les réactions du public. Ginsberg, qui revendique son homosexualité, est environné de dessins érotiques le représentant avec son ami Peter. Des vers au rythme nerveux - «affamés hystériques nus / se traînant à l'aube dans les rues nègres / à la recherche d'une furieuse piqûre" - sont suivis d'invectives à l'adresse de Moloch, monstre symbolisant le capitalisme et l'impérialisme américains - « Moloch dont l'âme est électricité et banque / Moloch dont la pauvreté est le spectre du génie » - tandis que fusent les cris, les sifflements du public.

56 À l'époque où paraît Hurlement, en automne 1956, Jack Kerouac a déjà écrit douze romans dont un seul a été édité. Sur la route, écrit en quatorze jours sur un rouleau de 
télétype, mais retravaillé sur la demande de son éditeur, sera publié le 5 septembre 1957.

\section{d'affirmation.}

Le Festin nu Burroughs prévient le lecteur que son livre est particulier, j'ai envie de dire à la limite du livre. Page 1, il écrit: "Je n'ai guère souvenir d'avoir rédigé les notes que l'on a publiées en langue anglaise sous le titre Naked Lunch." Pourtant, dans cette introduction d'ailleurs sous-titrée : "Témoignage à propos d'une maladie ", Burroughs écrit aussi, à propos du titre de son livre : «Il a exactement le sens de ses termes »; Le Festin nu c'est « cet instant pétrifié et glacé où chacun peut voir ce qui est piqué au bout de chaque fourchette."

61 Le livre oscille donc entre un hyperréalisme qui se concentre dans son titre et un flou qui envahit le corps de l'ouvrage. Plus précisément, le flou central est délimité par une introduction et une post-face qui, l'une et l'autre, donnent des clés pour la compréhension de la «maladie de la drogue ».

Que la drogue soit le seul sujet du Festin nu n'est pas une originalité - souvenons-nous des Confessions d'un mangeur d'opium anglais et des Paradis artificiels. Ce qui, en revanche, est nouveau, c'est le type de traitement littéraire de l'objet-drogue. Dans Le Festin nu, la drogue est, en effet, complètement dépourvue des raffinements de l'écriture. Dès lors, le corps à corps s'impose. C'est d'ailleurs en pensant au Festin nu que j'ai donné pour titre à ma communication : « la muse au corps à corps ».

63 J'ai suivi la structure de l'ouvrage pour mettre à jour ce corps à corps. L'introduction met à nu la séparation. Le corps de l'ouvrage fait état d'un envahissement. La post-face dévoile la nudité, la crudité de l'emprise.

"Je suis sorti de la Maladie, du "Mal" à l'âge de quarante-cinq ans, après quinze ans de toxicomanie ", écrit Burroughs au tout début de l'introduction. «J'ai consommé de la drogue par tous les moyens: je l'ai fumée, avalée, reniflée, injectée dans le réseau veines-peau-muscles, absorbée en suppositoires ». "Tout le monde en ferait autant", poursuit Burroughs, "parce que la drogue recèle la formule du virus diabolique, l'algèbre du besoin. »

65 «Nulle nécessité pour le vendeur d'améliorer ou de simplifier la marchandise, du moment où il amoindrit et simplifie le client ", écrit-il encore. Car le drogué a tout de la 
forme tronquée : «il reste en quelque sorte à l'écart, aux aguets, pendant que ses jambes de camé l'emmènent tout droit à la source de la came pour un nouveau plongeon. »

Ces formules fortes ici rassemblées, mais qui sont également concentrées dans l'introduction, rendent compte de l'accrochage à la drogue (on se drogue pour être accroché a écrit, en substance, Burroughs dans un ouvrage précédent, Junkie) et de l'impérieuse nécessité qui le gouverne. Dès lors "la renonce" est à la mesure de cet accrochage. «Le Festin nu est fatalement brutal, obscène et répugnant. La maladie et ses détails cliniques ne sont pas pour les estomacs délicats ", avertit Burroughs à la fin de l'introduction. Et pour bien marteler le propos, il indique dans un post-scriptum qu'il s'est désintoxiqué parce que ses fournisseurs lui avaient coupé les vivres.

67 La renonce s'est donc imposée en raison d'une contrainte externe, non d'une prise de conscience. "Trêve de boniments fatigués... le gars qui pratique l'héroïne n'ouvre pratiquement pas la bouche. » C'est bien d'un corps à corps dont il s'agit, durant lequel la séparation est forcément brutale.

Dans le corps de l'ouvrage règne une prolifération hybride. Les scènes d'hôpital souvent crues et plus encore désincarnées, à la limite de la science-fiction - côtoient les délires érotiques pervers ou comiques ainsi que les séances de consommation de drogue, sur un marché oriental, dans un coin de square de New York, dans une rue de Mexico.

69 C'est dans la post-face que Burroughs rend compte, après-coup, de cette prolifération, de cet envahissement: «le livre expulse ses pages dans toutes les directions... cris d'horreur et de passion, ethos et pathos et pataquès. » Ou encore : « on peut couper Le Festin nu à n'importe quel point d'intersection ».

Dans la post-face, Burroughs indique aussi qu'au-delà, ou en deçà de cette lecture au premier degré, une autre lecture exige le silence, parce que seul le silence peut répondre à l'héroïne : «silence de l'héroïne dans le silence en contrepoint des cellules assoiffées au petit matin ».

71 C'est au plus près du corps, dans le corps à corps, que surgissent alors les figures de l'emprise.

72 "Le singe cramponné au cou », " le singe que les drogués ont l'impression de porter en permanence sur le dos", c'est "le besoin fait monstre». Une telle figure peut décrocher de "l'algèbre du besoin " - autre formule de Burroughs - le temps d'une note d'humour : «assieds-toi, petit, je t'offre de la tarte aux myrtilles... une bouchée pour toi, une bouchée pour le singe qui te bouffe le dos, ça lui donne le poil luisant. » Mais elle dit surtout l'urgence du besoin : « garde ma valise un moment, gamin, il faut que j'aille faire pisser mon singe ». Mais elle montre surtout la crudité du corps à corps avec la drogue : « le singe qui est au fond de l'égout t'arrache l'âme pour qu'elle serve d'enseigne ».

73 «Les jours enfilés à la seringue » est une autre figure qui émerge, çà et là, du pot pourri - autre formule de Burroughs à prendre au pied de la lettre - du Festin nu. Figure percutante parce que brève. Figure lucide parce que sèche. Figure qui dit la répétition et la trajectoire de vie repliée sur le fil de la seringue. Figure qu'explicite Burroughs lorsqu'il parle " d'innombrables jouissances à froid».

74 On est aux antipodes - littéraires, bien sûr - des "étranges actions de grâce " de Baudelaire citant de Quincey : «Ô juste, subtil et puissant opium ». Autre époque, autre 
manière de transposer dans l'écriture le lien à la drogue. Chez Baudelaire, chez Burroughs, c'est parce que la drogue est un objet littéraire qu'elle a pu se dire dans des mots aussi forts que vrais ${ }^{1}$.

\section{NOTES}

1.. Bibliographie : BAUDELAIRE, Euvres complètes, Paris, Gallimard, Bibliothèque de la Pléiade, 1975 ; W. BURRoughs, Le Festin nu, Paris, Gallimard, 1964 ; J. JOHNSON, Personnages secondaires, Paris, Union générale d'éditions, 1996 ; M. KUNTZ, Les Toxicomanes, du goût de la drogue au goût de la contrainte, Paris, L'Harmattan, 1998 ; J. PIERROT, Merveilleux et fantastique. Une histoire de l'imaginaire dans la prose française du Romantisme à la Décadence (1830-1900), thèse de Lettres, Université de Lille III, 1975.

\section{RÉSUMÉS}

Cet article se veut exploration de deux époques où la drogue noua des liens serrés avec la littérature. Au milieu du XIX ${ }^{\mathrm{e}}$ siècle, scientifiques et littéraires se retrouvent au club des haschischins ; en 1860 naît une œuvre majeure, Les Paradis artificiels de Baudelaire, où la drogue est l'objet de comparaisons, de correspondances, de dialogues entre écrivains. Dans les années 50, le mouvement de la beat generation se cristallise dans quelques quartiers new-yorkais; dans Le Festin $n u$ de William Burroughs, parce que la drogue est complètement dépourvue des raffinements de l'écriture, le corps à corps s'impose.

This text deals with two different times when drugs were tightly related to literature. In the midst $19^{\text {th }}$ Century, scientists and writers met at the "Club des haschischins"; in 1860, Baudelaire publishes the Flowers of Evil, in which drug is a pretext of correspondences, comparisons and dialogs among writers. In the Fifties, the beat generation is set in New York. In Burroughs Naked Lunch, because drug is totally empty of any writing refinements, a struggle to the body replaces it. 